\title{
Crystal growth and structural studies of spinel ferrites
}

\author{
Jonas Sandemann* and Bo Brummerstedt Iversen \\ Department of Chemistry \& Interdisplinary Nanoscience Center (iNANO), Aarhus University, Denmark \\ *jonas@inano.au.dk
}

An important frontier in materials science is to understand, characterize and quantize disorder in inorganic materials and its relation to their properties. This requires deep knowledge of both the average structure and the defects present in the samples. Spinel-type compounds form a family of industrially relevant materials 1 that potentially exhibit both atomic and/or magnetic disorder.2, 3 Spinel ferrites, $\mathrm{AFe} 2 \mathrm{O} 4$, in particular have seen use in high-frequency applications due to their magnetism in conjunction with electrically insulating properties. The spinel structure consists of a distorted cubic closest packing of oxygen, in which 1/8 of the tetrahedral holes and $1 / 2$ of the octahedral holes are occupied by cations. The general formula is $\mathrm{AB}_{2} \mathrm{O}_{4}$, where $\mathrm{A}$ are divalent and $\mathrm{B}$ trivalent cations.

Single crystals larger than $1 \mathrm{~mm} 3$ of $\mathrm{ZnFe}_{2} \mathrm{O}_{4}$ and $\mathrm{NiFe}_{2} \mathrm{O}_{4}$ have been grown using the flux method. These were chosen as model spinel ferrites exhibiting the normal and inverse configuration, respectively, with the possibility of magnetic disorder studies in $\mathrm{ZnFe}_{2} \mathrm{O}_{4.4} \mathrm{X}$-ray fluorescence measurements confirmed a low degree of flux inclusions in the crystals, on the order of $0.1 \mathrm{wt} \%$.

Extensive diffraction data has been collected for initial benchmark structure determination, with synchrotron powder X-ray diffraction and single crystal X-ray diffraction being collected at SPring-8 in Japan, and single crystal neutron diffraction being collected at the Spallation Neutron Source at Oak Ridge National Laboratory. Initial data modelling shows some systematic discrepancies between the structural parameters obtained from the different sets of diffraction data. Rietveld modelling of the powder data gives lower lattice parameters than either single crystal method, which has been attributed to abnormal peak asymmetry caused by a nonsymmetric X-ray beam profile. The atomic displacement parameters obtained from the X-ray single crystal and powder data of $\mathrm{ZnFe} 2 \mathrm{O} 4$ differs both in magnitude and temperature dependence, the cause of which has not been identified yet.

The powder patterns of $\mathrm{NiFe}_{2} \mathrm{O}_{4}$ reveal left shoulders at reflections with miller indices that are all multiples of four, which could be related to the compound's magnetism.

The single crystal data show peak splitting indicating a degree of twinning on both spinel samples. Maximum entropy method analysis of the structure factors from the single crystal X-ray data showed no evidence of residual electron density at potential interstitial sites in the structure.

[1] N. Grimes, Physics in Technology, 1975, 6, 22.

[2] S. Sommer, E. D. Bøjesen, N. Lock, H. Kasai, J. Skibsted, E. Nishibori and B. B. Iversen, Dalton Transactions, 2020, 49, 13449-13461.

[3] K. Kamazawa, Y. Tsunoda, H. Kadowaki and K. Kohn, Physical Review B, 2003, 68, 024412.

[4] Y. Yamada, K. Kamazawa and Y. Tsunoda, Physical Review B, 2002, 66, 064401.

Keywords: Spinel, crystal growth, MEM, neutron, synchrotron 